Louisiana State University

LSU Digital Commons

Faculty Publications

Department of Physics \& Astronomy

$11-1-2007$

\title{
An extremely bright echo associated with SN 2002hh
}

D. L. Welch

McMaster University

Geoffrey C. Clayton

Louisiana State University

Amy Campbell

Louisiana State University

M. J. Barlow

University College London

Ben E.K. Sugerman

Space Telescope Science Institute

See next page for additional authors

Follow this and additional works at: https://digitalcommons.Isu.edu/physics_astronomy_pubs

\section{Recommended Citation}

Welch, D., Clayton, G., Campbell, A., Barlow, M., Sugerman, B., Meixner, M., \& Bank, S. (2007). An extremely bright echo associated with SN 2002hh. Astrophysical Journal, 669 (1), 525-533. https://doi.org/10.1086/ 521542

This Article is brought to you for free and open access by the Department of Physics \& Astronomy at LSU Digital Commons. It has been accepted for inclusion in Faculty Publications by an authorized administrator of LSU Digital Commons. For more information, please contact ir@lsu.edu. 


\section{Authors}

D. L. Welch, Geoffrey C. Clayton, Amy Campbell, M. J. Barlow, Ben E.K. Sugerman, Margaret Meixner, and S. H.R. Bank 


\title{
AN EXTREMELY BRIGHT ECHO ASSOCIATED WITH SN 2002hh \\ D. L. Welch, ${ }^{1}$ Geoffrey C. Clayton, ${ }^{2,3}$ Amy Campbell, ${ }^{2}$ M. J. Barlow, ${ }^{4}$ Ben E. K. Sugerman, ${ }^{5,6}$ Margaret Meixner, ${ }^{5}$ and S. H. R. Bank ${ }^{3,7}$ Received 2006 October 30; accepted 2007 July 10
}

\begin{abstract}
We present new, very late time optical photometry and spectroscopy of the interesting Type II-P supernova SN 2002hh, in NGC 6946. Gemini/GMOS-N has been used to acquire visible spectra at six epochs between 2004 August and 2006 July, following the evolution of the SN from age 661 to 1358 days. Few optical spectra of Type II supernovae with ages greater than $1 \mathrm{yr}$ exist. In addition, $g^{\prime}, r^{\prime}$, and $i^{\prime}$ images were acquired at all six epochs. The spectral and photometric evolution of SN 2002hh has been very unusual. Measures of the brightness of this SN, both in the $R$ and $I$ bands, as well as in the $\mathrm{H} \alpha$ emission flux, show no significant fading over an interval of nearly 2 years. The most straightforward explanation for this behavior is that the light being measured comes not only from the SN itself but also from an echo off nearby dust. Echoes have been detected previously around several SNe, but these echoes, at their brightest, were $\sim 8$ mag below the maximum brightness of the SN. At $V \sim 21 \mathrm{mag}$, the putative echo dominates the light of SN 2002hh and is only $\sim 4$ mag below the outburst's peak brightness. There is an estimated 6 mag of total extinction in $V$ toward SN $2002 \mathrm{hh}$. The proposed explanation of a differential echo/SN absorption is inconsistent with the observed $B V R I$ colors.
\end{abstract}

Subject headings: dust, extinction — scattering — supernovae: general

\section{INTRODUCTION}

The condensation of dust in supernova (SN) ejecta is not well constrained by observations, since the detection of newly formed dust in young $\mathrm{SNe}$ in nearby galaxies has been extremely rare. Signatures of condensing dust can be observed in nearby Type II SNe approximately 1-2 yr after core collapse. There are three strong indications of the formation of dust: (1) a sudden decrease in continuum brightness in the visible due to increased dust extinction, (2) an accompanying development of an infrared excess arising from dust grains absorbing higher energy photons and reemitting them in the infrared, and (3) the development of asymmetric, blueshifted emission-line profiles (e.g., Sugerman et al. 2006). This combination of observable effects can be best explained by dust forming within the expanding ejecta and preferentially extinguishing emission from the far-side (hence redshifted) gas, which also results in a shift of the centroid of the emission-line profiles to the blue, optical dimming, and mid-infrared brightening (Lucy et al. 1989, 1991). Asymmetric emission-line profiles have been seen in SNe 1987A, 1998S, 1999em, 2003gd, and possibly 2004et (e.g., Danziger et al. 1991; Gerardy et al. 2000; Elmhamdi et al. 2003; Pozzo et al. 2004; Sugerman et al. 2006; Sahu et al. 2006). In the sample of seven supernovae with such spectra, dust appears to have formed in the ejecta of at least five. Even within this small sample the dust formation times range from near 300 days to 550 days.

\footnotetext{
1 Department of Physics and Astronomy, McMaster University, Hamilton, ON, L8S 4M1 Canada; welch@physics.mcmaster.ca.

2 Department of Physics and Astronomy, Louisiana State University, Baton Rouge, LA 70803; gclayton@fenway.phys.lsu.edu, campbell@theory.phys.lsu.edu.

${ }^{3}$ Maria Mitchell Observatory, 4 Vestal Street, Nantucket, MA 02554.

4 Department of Physics and Astronomy, University College London, Gower Street, London WC1E 6BT, UK; mjb@star.ucl.ac.uk.

5 Space Telescope Science Institute, 3700 San Martin Drive, Baltimore, MD 21218; meixner@stsci.edu.

6 Department of Physics, Goucher College, 1021 Dulaney Valley Road, Baltimore, MD 21204; ben.sugerman@goucher.edu.

7 Department of Physics, UMBC, 1000 Hilltop Circle, Baltimore, MD 21250; sbank1@umbc.edu.
}

SN 2002hh was discovered in NGC 6946 on 2002 October 31 (Li 2002). With the subsequent discovery of SN 2004et, there have now been eight SNe discovered in NGC 6946 in less than a century. A spectrum of SN 2002hh, obtained on 2002 November 2 , showed a broad but very low contrast $\mathrm{H} \alpha$ emission and absorption features with a very red, nearly featureless continuum, indicating a very young Type II SN (Filippenko et al. 2002). Optical imaging shows extensive dust obscuration in this region of NGC 6946 and Meikle et al. (2002) estimate a total absorption of $A_{V} \sim 6.1 \mathrm{mag}$ toward SN $2002 \mathrm{hh}$ of which about $1 \mathrm{mag}$ is Galactic foreground obscuration. This estimate was made by comparing the observed $J-K_{S}$ color to template IR light curves (Mattila \& Meikle 2001) and is supported by the strong interstellar Na I D absorption seen in the spectrum (Filippenko et al. 2002). CO emission was seen in $K$-band spectra of SN 2002hh in the first 6 months after outburst (Pozzo et al. 2004). The light curve of SN 2002hh shows it to be a Type II-P, where the SN remains at or near maximum brightness for $\sim 3$ months after the explosion (Pozzo et al. 2006).

Thermal emission from dust in or near SN 2002hh was detected at mid-infrared wavelengths from 590 to 994 days by the Spitzer Space Telescope and confirmed by higher angular resolution Gemini/Michelle observations (Barlow et al. 2005; Meikle et al. 2006). An optically thick dust shell has been inferred, having a mass of $0.04-0.15 M_{\odot}$ and suggesting a massive $\mathrm{M}$ supergiant or luminous blue variable precursor. The inner radius of the shell is estimated to be $\sim 1 \times 10^{17}$ to $\sim 1 \times 10^{18} \mathrm{~cm}$ (Barlow et al. 2005 ; Meikle et al. 2006). The highest velocity gas from the SN 2002hh explosion will not reach this circumstellar dust for approximately $28 \mathrm{yr}$ (Meikle et al. 2006). Barlow et al. (2005) could not rule out the formation of new dust in the ejecta of SN $2002 \mathrm{hh}$, since any mid-infrared emission from new dust would be swamped by emission from the existing circumstellar dust, which would occupy a far larger solid angle.

Pozzo et al. (2006) first suggested the existence of an IR echo in the $J H K$ bands around SN $2002 \mathrm{hh}$ at early epochs up to 314 days. Meikle et al. (2006) found that the mid-IR flux declined by about $10 \%$ from 590 to 994 days. They suggested that most of the mid-IR 


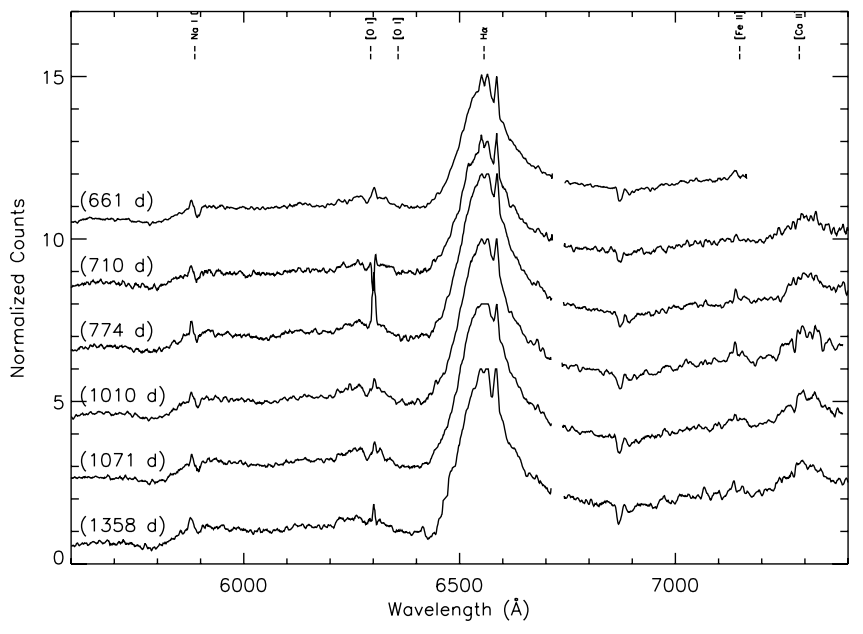

FIG. 1.-New Gemini/GMOS spectra of SN 2002hh from day 661 to day 1358. For display purposes, the narrow emission lines from NGC 6946 have been truncated.

flux may have come from dust in the star formation region associated with the SN 2002hh precursor. They found no evidence for new dust condensing in the ejecta, nor did they attribute the bulk of the emission to heated circumstellar material. The small declining component of the IR flux is attributed to an IR echo. An echo around SN 2002hh has been confirmed in the optical by B. E. K. Sugerman et al. (2007, in preparation), who have found a small but well-resolved circular scattered-light echo in visible light roughly $0.18^{\prime \prime}$ in diameter at the astrometric position of the supernova. In this paper we present a series of new, late-time optical spectra and photometry of the Type II-P SN 2002hh that were obtained to look for evidence of dust formation in its ejecta.

\section{OBSERVATIONS}

Spectroscopy was obtained using the Gemini North telescope and the GMOS-N instrument during semesters 2004B, 2005B, and 2006A. Three spectra of duration $900 \mathrm{~s}$ each were obtained on 2004 August 20. These were obtained in long-slit mode with a slit orientation of $190^{\circ}$ in order to make use of a bright guide star. A $0.75^{\prime \prime}$ slit width was used for the observations with the B600G5303 grating in first order. The central wavelengths of the spectra were 5720,5700 , and $5680 \AA$, respectively, to ensure that the two gaps between GMOS-N's three CCD chips could be bridged. A $2 \times 2$ binning of the CCD pixels in the low gain setting was employed.

Two additional epochs for SN 2002hh in 2004 were obtained using Director's Discretionary (DD) time. On 2004 October 8, three spectra, each of $720 \mathrm{~s}$ duration, were obtained with a setup identical to that described above. The observations had central wavelengths of 5970, 5950, and $5930 \AA$, respectively. These longer central wavelengths were selected in order that the $[\mathrm{Ca}$ II] $\lambda \lambda 7291,7324$ lines would be recorded. A second set of spectra were obtained on 2004 December 11 UT. Each had a duration of 720 s and had central wavelengths of 5970, 5950, and $5930 \AA$, respectively.

Two epochs of spectra were obtained in 2005. The configuration of GMOS-N was identical to that above. The first epoch was obtained on 2005 August 4. Each exposure had a duration of $900 \mathrm{~s}$ to reduce the effect of fading of the SN on the signal-tonoise ratio of the spectra and had central wavelengths of 5970, 5950 , and $5990 \AA$, respectively. The second epoch was obtained on 2005 October 4 , and each spectrum then also had an exposure of 900 s. The central wavelengths were 5970, 5950, and $5990 \AA$, respectively.

The final epoch of spectra reported in this paper were obtained under an identical GMOS-N configuration on 2006 July 18. Each had a duration of $900 \mathrm{~s}$ and had central wavelengths of 5970, 5950, and $5990 \AA$, respectively.

All spectra had adjacent GCal flat exposures taken prior to changes to new central wavelengths. $\mathrm{CuAr}$ spectra used to calibrate the pixel-to-wavelength transformation were obtained during daytime. A single set of bias frames was used to reduce all of the spectra. The spectra from each individual night were averaged and normalized.

Spectra were reduced using IRAF version 2.12.2a and version 1.8 of the IRAF external package gemini. The usual pattern of reduction tasks gbias, gsflat, gsreduce, gswavelength, gstransform, gsskysub, and gsextract was employed. Sky subtraction regions were identified by eye and usually incorporated about 50 pixels on either side of the SN spectrum itself. Observations were not fluxed due to the lack of photometric conditions on several of the nights and also due to the primary goal of recording line profile variations. The radial velocity of NGC 6946 is $+48 \mathrm{~km} \mathrm{~s}^{-1}$. Emission lines from incompletely subtracted $\mathrm{H}$ in regions in the SN 2002hh spectra allow us to register the supernova to the local standard of rest in the galaxy. The spectra are shown in Figure 1 and are summarized in Table 1. (The spectra in Fig. 1 have not been dereddened.) We adopt 2002 October 29 as the explosion epoch (Pozzo et al. 2006).

The position of the peak of the $\mathrm{H} \alpha$ emission line is poorly determined due to the contamination of the $\lambda \lambda 6548-6584$ [N II] doublet and the $\lambda 6563 \mathrm{H} \alpha$ line from the $\mathrm{H}$ II region. To gauge the change in line position and symmetry, we instead measured the wavelength at which the ascending and descending portions of the line profile reached $50 \%$ of their peak values. Prior to making this measurement, a linear continuum slope correction was applied based on the average intensity levels in the $\lambda \lambda 6240-6260$ and 6940-6960 regions of the spectra. The intensity of the $\mathrm{H} \alpha$ line changes rapidly through the $50 \%$ points, ensuring precise estimates

TABLE 1

Half-Intensity H $\alpha$ Wavelengths and Photometry for SN 2002hh

\begin{tabular}{|c|c|c|c|c|c|}
\hline Observation Date (UT) & $\begin{array}{l}\text { Age } \\
\text { (days) }\end{array}$ & $\begin{array}{c}50 \% \mathrm{H} \alpha \lambda_{\text {asc }} \\
(\AA)\end{array}$ & $\begin{array}{c}50 \% \mathrm{H} \alpha \lambda_{\mathrm{des}} \\
(\AA)\end{array}$ & $\begin{array}{c}R^{\mathrm{a}} \\
(\mathrm{mag})\end{array}$ & $\begin{array}{c}I^{\mathrm{a}} \\
(\mathrm{mag})\end{array}$ \\
\hline 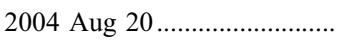 & 661 & 6499.9 & 6618.8 & 19.87 & 18.98 \\
\hline 2004 Oct 8 & 710 & 6497.2 & 6620.8 & 19.91 & 19.01 \\
\hline 2004 Dec $11 \ldots \ldots \ldots \ldots \ldots \ldots \ldots$ & 774 & 6499.6 & 6625.5 & 19.97 & 19.13 \\
\hline 2005 Aug 4 & 1010 & 6498.9 & 6619.6 & 20.15 & 19.35 \\
\hline 2005 Oct $4 \ldots \ldots \ldots \ldots \ldots$ & 1071 & 6500.2 & 6623.2 & 20.15 & 19.40 \\
\hline 2006 Jul 18 & 1358 & 6497.9 & 6621.1 & $\ldots$ & 19.60 \\
\hline
\end{tabular}

${ }^{\text {a }}$ The $R$ and $I$ magnitudes were converted from the observed $g^{\prime}, r^{\prime}$, and $i^{\prime}$ instrumental magnitudes. See text. 


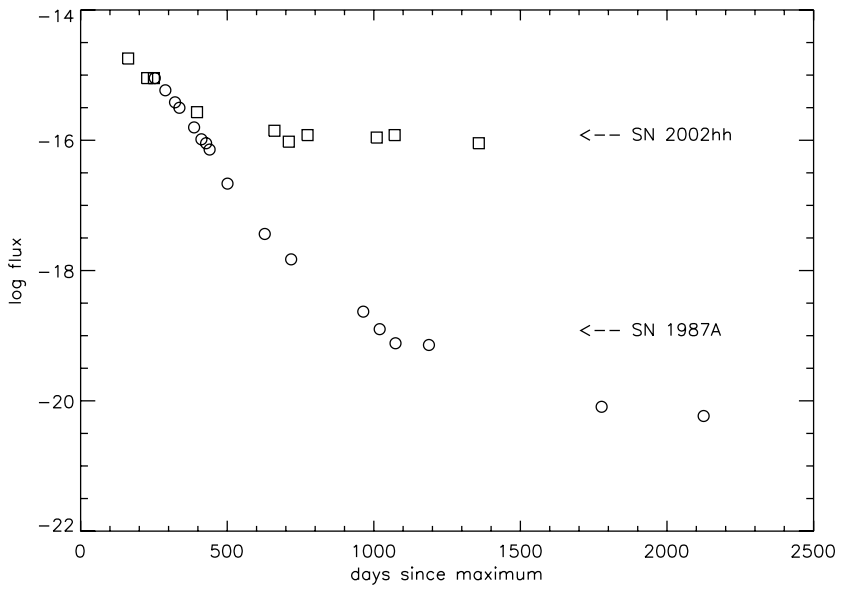

FIG. 2.-Measures of the peak counts in $\mathrm{H} \alpha$ in SN 1987A (circles) and SN $2002 \mathrm{hh}$ (squares) plotted against time since maximum light.

for the ascending and descending half-peak wavelengths. In Table 1 we list, from left to right, the UT observation date, the age of the supernova, the wavelength at which the ascending portion of the $\mathrm{H} \alpha$ line reached $50 \%$ of its peak value, and the corresponding position on the descending wing. The uncertainty in these wavelengths is $\pm 2 \AA$, based on the repeatability of the number for each of the three or four spectra obtained on a given observation date.

On each of the six epochs described above, SN 2002hh was also observed in imaging mode with GMOS-N in the $g^{\prime}, r^{\prime}$, and $i^{\prime}$ bands. Exposure times were $60 \mathrm{~s}$. The instrumental $g^{\prime}, r^{\prime}$, and $i^{\prime}$ magnitudes were converted to standard Johnson-Cousins $R$ and $I$ magnitudes using the photometric sequence from Table 1 of Pozzo et al. (2006). The standard stars that fell on the central chip of GMOS-N (the chip on which SN 2002hh was imaged) were used for this purpose.

A simultaneous least-squares solution involving common color terms but floating zero points for each night was used to transform the instrumental $g^{\prime}, r^{\prime}$, and $i^{\prime}$ ALLFRAME magnitudes to the $V$, $R$, and $I$ sequence from Pozzo et al. (2006). The uncertainties in the $V, R$, and $I$ transformations thus derived were $0.033,0.014$, and $0.010 \mathrm{mag}$, respectively. All transformations were confirmed to be linear, with the bulk of the higher uncertainty in the $V$ transformation being due to the residuals of two stars of intermediate color, 13 and 14 .

The transformations were then inverted to allow the estimation of the $V, R$, and $I$ magnitudes from the instrumental $g^{\prime}, r^{\prime}$, and $i^{\prime}$ magnitudes. The derived transformations are

$$
\begin{aligned}
& V=0.7111 g^{\prime}-0.0829 r^{\prime}+0.3728 i^{\prime}+\text { zero point } \\
& R=0.0000 g^{\prime}+0.7540 r+0.2264 i^{\prime}+\text { zero point } \\
& I=0.0000 g^{\prime}-0.2786 r^{\prime}+1.2531 i^{\prime}+\text { zero point. }
\end{aligned}
$$

We were not able to provide $V$ magnitudes for SN 2002hh because the supernova light was too confused with other nearby sources of similar brightness in every image taken in the $g^{\prime}$ bandpass. On 2006 July 18, SN $2002 \mathrm{hh}$ could not be measured accurately in the $r^{\prime}$-band image for similar reasons, but if it is assumed that the color of the SN was similar to that of the Pozzo standard number 5 , then $I=19.60 \mathrm{mag}$. (The small coefficient of the $r^{\prime}$ magnitude in the transformation to I makes the transformation reasonably robust to reasonable values for the color.) The measured $R$ and $I$ magnitudes of SN 2002hh for the six epochs are listed in Table 1 and plotted in Figure 2.

\section{DISCUSSION}

\subsection{Spectra}

Pozzo et al. (2006) observed SN 2002hh in the optical and near-IR from day 3 to 397 . Optical spectra, including $\mathrm{H} \alpha$ and [O I ] $\lambda \lambda 6300,6364$, were obtained at seven epochs. Pozzo et al. commented that, in general, the spectra of SN 2002hh resemble those of SN 1987A at similar epochs. In particular, SN 1987A and SN 2002hh both showed P Cygni profiles in $\mathrm{H} \alpha$ and $\mathrm{Na}$ I D at early times (Phillips \& Williams 1991). The biggest difference between the SN 2002hh and SN 1987A was the strength of the [Ca II] $\lambda \lambda 7291,7323$ and the Ca II near-infrared triplet, which was much stronger in SN 1987A (Pozzo et al. 2006). Their last spectrum, obtained on day 397, shows no evidence for a developing asymmetry due to dust forming in the ejecta.

Figure 1 shows the new spectra obtained at an additional six epochs, which extend the spectral coverage to day 1358. None of the new spectra reveal an asymmetry in $\mathrm{H} \alpha$ similar to that which developed in SN 1987A around day 526 and which is believed to have indicated dust formation (see Fig. 7 of Phillips \& Williams 1991). The H $\alpha$ profile in SN 2002hh never completely lost its P Cygni shape, having a broader red wing than a blue wing (Duschinger et al. 1995). Not only is there no development of a blue asymmetry, but also the FWHM of $\mathrm{H} \alpha$ has increased in the day 661-1358 spectra compared to the last epoch of Pozzo et al. (2006) at day 397 without any statistically significant shift in line centroid. No increase in the opacity in the red wing of the line is seen in SN 2002hh. In fact, the red and blue wings both become broader between day 397 and 661. No such broadening of $\mathrm{H} \alpha$ was seen in SN 1987A (Phillips \& Williams 1991). As seen in Table 1, the half-power points of $\mathrm{H} \alpha$ show no sensible evolution of either position over the course of the observations reported here.

The fact that the $\mathrm{H} \alpha$ emission-line profile has not developed a blue asymmetry but rather has retained its P Cygni shape and broadened since day 397 may be due to the presence of an echo around SN 2002hh. It is possible that dust formation in $2002 \mathrm{hh}$ is perhaps hidden or its interpretation confounded by scattered light from an echo resulting in a systematic error in the estimated age of the spectral features in the optical (Meikle et al. 2006). However, Pozzo et al. (2006) note that the spectrum of SN 2002hh obtained on day 397 has developed a strong blue continuum, indicating that the echo may have been affecting the light from SN $2002 \mathrm{hh}$ even at this early epoch.

Since SN 2002hh is a Type II-P, it stayed near maximum brightness for $\sim 3$ months. Although there is a gap in the photometry of SN 2002hh from day 43 to 166, its light-curve behavior was consistent with both SN 1987A and SN 1999em (Pozzo et al. 2006). Any SN light echo pulse width will be quite broad, since it will contain an integral of the SN light over the first 3 months. Van Dyk et al. (2006) estimated a pulse width of $\sim 43$ days in the $B$ band and $\sim 138$ days in the $R$ band for the Type II-P SN 2003gd. The pulse width of SN $2002 \mathrm{hh}$ is likely to be on the same order as the length of the plateau phase of the light curve, $\sim 100$ days. The echo spectrum seen at late times would have been an average of the SN spectrum over this period. Pozzo et al. (2006) reported spectra from SN $2002 \mathrm{hh}$ at days 4, 8, and 44. At day 44, the lines in the SN spectrum had already taken on the P Cygni profile shape that was seen all the way out to day 1358 .

The late-time Gemini/GMOS spectra do resemble the Pozzo et al. (2006) day 44 spectrum of SN 2002hh. The day 44 spectrum and the new late-time (day 661-1358) spectra presented here are all broader than the last Pozzo et al. epoch at day 397. The $\mathrm{H} \alpha$ emission profiles from the six Gemini/GMOS epochs spanning over 
700 days are almost identical and show no significant profile evolution with time as seen in Figure 1. We find that the extrema of the $\mathrm{H} \alpha$ wings indicate an expansion velocity between 11,000 and $13,000 \mathrm{~km} \mathrm{~s}^{-1}$ for our observed epochs, where the uncertainty is dominated by the assumption of the continuum locations. The FWHM of all six epochs are $\sim 5600 \mathrm{~km} \mathrm{~s}^{-1}$. This is significantly broader than the profiles from days 162 to 397, which have FWHM of $\sim 4500 \mathrm{~km} \mathrm{~s}^{-1}$ (Pozzo et al. 2006). No spectra were taken between days 44 and 162, but the day 44 spectrum was in the middle of the time period of the light pulse, which we believe is being scattered toward us in the echo. These late-time $\mathrm{H} \alpha$ profiles, broader than the previously observed epoch (day 397), support the interpretation that the late-time spectrum is dominated by the echo.

To investigate the contribution from the echo, the counts from $\mathrm{SN} 2002 \mathrm{hh}$ in the $\mathrm{H} \alpha$ emission-line profiles were examined. In Figure 2, the peak counts in $\mathrm{H} \alpha$ in the Gemini/GMOS spectra from all six epochs are plotted together, scaled to their exposure times, along with the epochs observed by Pozzo et al. (2006). The spectra could not be fluxed, since no standards were observed, but they were acquired with the same slit and the same setup and at similar air masses during each observation. The counts should be roughly representative of the relative fluxes, taking into account the uncertainties $(\sim 30 \%)$ introduced by differences in seeing and transparency. In particular, the lowest count rates were associated with the worst seeing, which occurred on days 710 and 1358. This comparison in Figure 2 shows that there is no evidence for significant fading of the peak flux in $\mathrm{H} \alpha$ over the period from day 661 to 1358. We also plot the evolution of SN 1987A's scaled peak H $\alpha$ flux (Caldwell et al. 1993; the SUSPECT supernova spectrum database) in Figure 2. If SN 2002hh had faded in a manner similar to SN 1987A, then from day 397 (Pozzo et al. 2006) to day $1358, \mathrm{H} \alpha$ should have faded by a factor of $\sim 10^{4}$. However, SN 2002hh's H $\alpha$ flux drops by only a factor of 2 from 397 to 1358. Since SN 2002hh is a Type II SN, its massive precursor star likely was located in a star formation region with significant $\mathrm{H} \alpha$ flux. We have decomposed the narrow $\mathrm{H}$ II region emission lines and compared their flux to the broad SN H $\alpha$ emission. On day 661, the narrow emission lines made up only $16 \%$ of the flux. So most of the flux is from the $\mathrm{SN}$, which is distinguished in any case by its very broad $\mathrm{H} \alpha$ emission up to day 1358 . The fact that the broad $\mathrm{H} \alpha$ emission profile can be seen and measured even at day 1358 means that we are still measuring SN 2002hh without significant contamination from other sources.

\subsection{Photometry}

The latest published $V$-band photometry of SN 2002hh, reported by Pozzo et al. (2006), was on day 269 - too early to expect to see evidence of fading due to dust formation in the ejecta and also before the echo became noticeable in the optical. Pozzo et al. (2006) detected a $K-L^{\prime}$ excess that developed in the photometry of SN $2002 \mathrm{hh}$ between days 200 and 314. This may indicate the presence of an infrared echo, presumably produced by early-time heating of the circumstellar dust by the SN. Meikle et al. (2006) found no evidence for new dust condensing in the ejecta and suggested that most of the mid-infrared flux may have come from dust in the star formation region associated with SN $2002 \mathrm{hh}$ 's precursor. They also found that the mid-infrared flux declined by about $10 \%$ from day 590 to 994 . The small declining component of the infrared flux was attributed to an infrared echo.

The brightness of the echo at optical wavelengths was highly unexpected; the late-time observed brightness of SN 2002hh in the $V$ band was only 4 mag fainter than the light-curve peak of
$V=17.2$ mag (Pozzo et al. 2006; B. E. K. Sugerman et al. 2007, in preparation). The photometry is also consistent with the lack of fading seen in the $\mathrm{H} \alpha$ emission shown in Figure 2. Typically, one would expect a light echo to appear fainter than the SN maximum by 8 mag or more (Patat 2005).

The fading of SN $2002 \mathrm{hh}$, from its maximum brightness of $R \sim 15.5$ mag and $I \sim 14.3$ mag leveled off around $R \sim 20$ mag and $I \sim 19$ mag sometime between day 269, the last Pozzo et al. (2006) data point, and day 661, the first data point obtained here. This behavior is very different from the SN 1987A light curve that is scaled and plotted for comparison in Figure 3. If SN 2002hh had behaved like SN 1987A, it would have been about 3-4 mag fainter in the $R$ and $I$ bands than observed on day 661 . SN $2002 \mathrm{hh}$ seems to have faded slowly from $I \sim 19.0$ to 19.6 mag between days 661 and 1358. By day 1358, SN 2002hh was $\sim 9$ mag too bright at $I$ when compared to SN 1987 A.

Observations of SN 2002hh in the optical were made with the High-Resolution Camera (HRC) of the Advanced Camera for Surveys (ACS) aboard the Hubble Space Telescope (HST) on 2005 September 17, 2006 April 23, and 2006 November 18, respectively, that is, on days 1054, 1272, and 1481 after maximum. As will be fully presented in B. E. K. Sugerman et al. (2007, in preparation), these observations reveal a small but well-resolved circular scattered-light echo roughly $0.18^{\prime \prime}$ in diameter at the astrometric position of the supernova. At an estimated distance of $5.9 \mathrm{Mpc}$, this implies that the scattering dust is roughly 12 lt-yr in front of the SN. The integrated fluxes in F435W, F606W, and F814W from the first epoch roughly convert to Johnson-Cousins $B, V$, and $I$ magnitudes of $24.3,21.4$, and $19.5 \mathrm{mag}$, respectively (Sirianni et al. 2005). The $V$-band brightness decreased by only a few hundredths of a magnitude in the subsequent two epochs. These measurements are fully consistent with ours and show that SN 2002 hh had still faded by only $\sim 4$ mag at $V$ from its maximum brightness by day 1481 .

By day 661, a typical Type II-P SN with no echo will have faded $\sim 7.5$ mag below its maximum brightness. Normally, an optically thin dust echo will grow brighter as the optical depth of the dust increases (Patat 2005). Patat's sheet model $(R=200$, $\Delta R=50$ ), assuming dust with $\tau=0.03$, will produce an echo with a brightness $\sim 11.75$ mag below the SN maximum, while dust with $\tau=2.5$ will produce an echo with a brightness only $\sim 6.75$ mag below the $\mathrm{SN}$ maximum. If multiple scattering is taken into account, as it must be when $\tau>1$, then $\tau=2.5$ will produce an echo with a brightness $\sim 9.25$ mag below the $\mathrm{SN}$ maximum or 3 mag fainter than in the single-scattering model.

There has been significant discussion in the literature regarding the nature of the dust along the direct line of sight to SN 2002hh. Pozzo et al. (2006) reconsidered the extinction for SN 2002hh in some detail and concluded that a line-of-sight dust pocket containing atypically small dust grains contributed to a total $A_{V}=$ $5.3 \mathrm{mag}$ as a result of a two-component dust model $\left(A_{V}=3.3 \mathrm{mag}\right.$ and $R_{V}=3.1 \mathrm{mag}$ plus $A_{V}=1.7 \mathrm{mag}$ and $\left.R_{V}=1.1 \mathrm{mag}\right)$. It is, a priori, rather unlikely that there is dust in NGC 6946 with $R_{V}=$ $1.1 \mathrm{mag}$. Another possible explanation is that there is a mismatch between the intrinsic spectral energy distribution (SED) of SN $2002 \mathrm{hh}$ and that of SN $1999 \mathrm{em}$, which was used as a lightly reddened template. Wang (2005) alternatively suggested that light scattered from circumstellar dust near the SN will cause apparently small values of $R_{V}$. It should be noted that in Wang's model the inner radius of the shell is at $10^{16} \mathrm{~cm}$, whereas Pozzo et al. (2006) suggest that dust within $10^{17} \mathrm{~cm}$ of the SN would be evaporated. The apparently low value for $R_{V}$ could also be caused by combining dust with two different values of $R_{V}$ (McGough et al. 


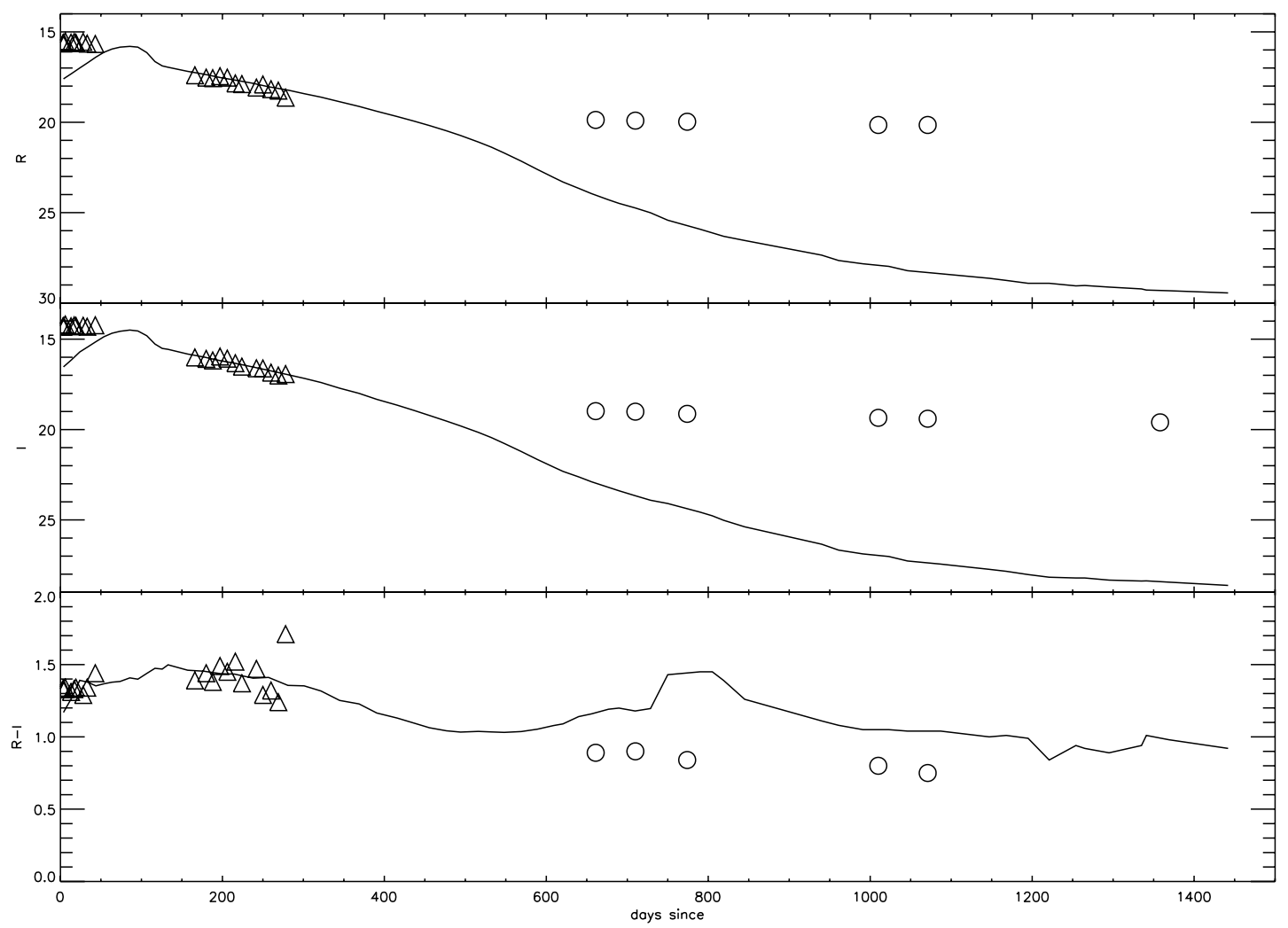

FIG. 3.-Light curve of SN 2002hh plotted against a scaled light curve from SN 1987A (Hamuy \& Suntzeff 1990; Walker \& Suntzeff 1991; solid line). The upper two panels show the observed $R$ and $I$ photometry, while the lower panel show the $R-I$ colors. The SN $1987 \mathrm{~A} R-I$ colors have been adjusted assuming $A_{V}=5$ mag and $R_{V}=3.1$. The SN 2002hh photometry is from Pozzo et al. (2006; triangles) and from this study (circles).

2005). Nevertheless, the possibility of anomalous dust must be seriously considered when modeling the echo in SN $2002 \mathrm{hh}$.

In one possible scenario, the extreme brightness of the $\mathrm{SN}$ $2002 \mathrm{hh}$ echo can be explained if the optical depth is much greater ( $\sim 6 \mathrm{mag}$ ) along the line of sight to the SN than in other nearby directions ( $\lesssim 1 \mathrm{mag}$ ). The SN light from the echo could skirt the large dust optical depth situated directly in front of SN 2002hh and be bright by comparison (see Fig. 1 of Patat et al. 2006.) The observed light curve and spectral evolution could be accounted for by the proposed differential total absorption.

Using the time-integrated spectrum of SN 1999em, reddened, as described by Pozzo et al. (2006) to reproduce the expected light pulse from SN 2002hh, the predicted observed colors for the SN 2002hh echo are $B-V=2.7 \mathrm{mag}, V-I=3.1 \mathrm{mag}$, and $R-I=1.3 \mathrm{mag}$. These are consistent with the colors actually observed for SN 2002hh at early epochs (Pozzo et al. 2006).
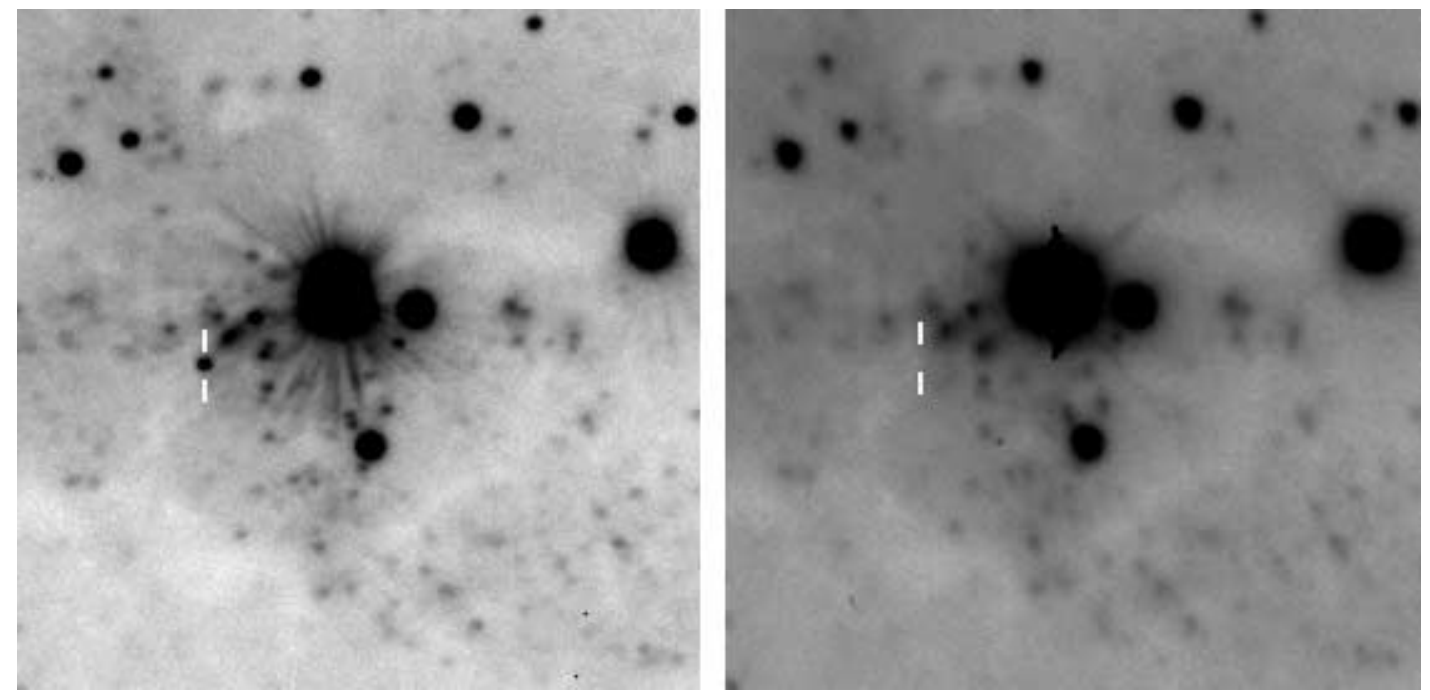

FIG. 4.-Left, Gemini/GMOS $r^{\prime}$ image taken on 2004 August 20; right, $R$-band image taken with the preoutburst CFHT CFH12K image on 2000 September 27. The

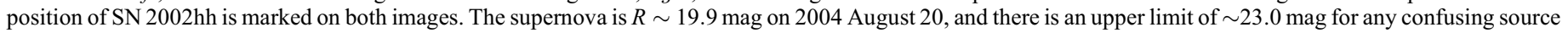
at the position of SN $2002 \mathrm{hh}$ on 2000 September 27 . The field is $40^{\prime \prime} \times 40^{\prime \prime}$. North is up, and east is to the left. 


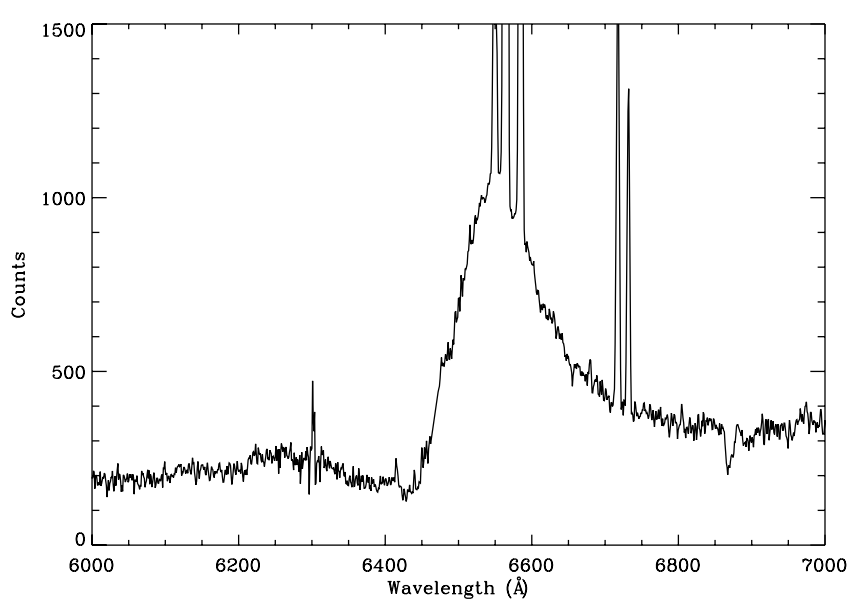

FIG. 5.-Gemini/GMOS spectrum of SN 2002hh taken on 2006 July 18. The plotted spectrum is the average of three individual spectra taken on the same date. The ratios of the peak line emission to the continuum on either side of the $\mathrm{H} \alpha$ line are very similar to those seen in the spectrum taken of SN 2002hh on day 44

However, at late epochs ( $>600$ days), when the light of SN 2002hh was apparently dominated by the echo light, the colors were $B-$ $V \sim 2.6 \mathrm{mag}, V-I \sim 1.8 \mathrm{mag}$, and $R-I \sim 0.8 \mathrm{mag}$. The $V-I$ and $R-I$ colors are significantly bluer than the observed earlytime colors of SN $2002 \mathrm{hh}$ however, these colors are reasonably consistent with the reddened supernova light scattering from Galactic-type dust with a modest optical depth $A_{V} \sim 0.5$ (Sugerman 2003). In this scenario, the echo light passes through the same dust that is reddening the directly transmitted light from SN 2002hh and then passes through and is scattered off dust lying nearby, which also adds a small amount of reddening.

No line-of-sight sources that might contaminate the light curve and spectroscopy of SN 2002hh are seen in the shifted and combined sum of three $120 \mathrm{~s} R$-band images $(550745 \mathrm{o}, 550746 \mathrm{o}$, and $550747 \mathrm{o}$ ) taken on UT 2000 September 27 prior to outburst and shown in Figure 4. The seeing and depth of the combined image is very similar to our Gemini $r^{\prime}$ images and allows us to constrain the precursor to be fainter than $R=23.0$ (or 7.5 mag fainter than maximum light). To further clarify the relative strength of the continuum and $\mathrm{H} \alpha$ line at late times, the unfluxed but sky-subtracted Gemini/GMOS spectrum for SN 2002hh is shown in Figure 5. The ratio of the peak $\mathrm{H} \alpha$ to blue-side continuum is 5.0, and the ratio of $\mathrm{H} \alpha$ to red-side continuum is 2.9 . These numbers are very similar to their day 44 counterparts of 4.75 and 2.7 (Pozzo et al. 2006), respectively, and fully consistent with the light echo explanation for the late-time photometry and spectroscopy.

We note that the appearance of a resolved echo clearly indicates the dominance of the echo light above the local background. In an aperture surrounding the echo in F606W, the sky value is $2.4 \times$ $10^{-3}$ (counts s$^{-1}$ pixel $^{-1}$ and in F658N it is $7.4 \times 10^{-4}$ counts s $^{-1}$ pixel $^{-1}$. This compares to peak values of the echo of 0.73 counts s ${ }^{-1}$ pixel $^{-1}$ in F606W and $9.2 \times 10^{-2}$ in F658N. In other words, the peak surface brightness of the echo is 300 times brighter than the background in F606W and 125 times brighter in F658N.

While the bluer $V-I$ and $R-I$ colors are consistent with the scenario in which the echo light is significantly less reddened than the direct SN light, the $B-V$ color measured by B. E. K. Sugerman et al. (2007, in preparation) is much too red. SN $2002 \mathrm{hh}$ is quite faint in the $B$ band and this measurement has a large uncertainty ( $>0.5 \mathrm{mag})$, so the $B-V$ color could be bluer than reported here. If the these colors are based solely on a less reddened path, then the $V-I$ and $R-I$ colors imply that the echo light passes through dust with an $A_{V}$ that is 2-2.5 mag less than the direct light from the SN. This scenario would serve to explain at least some of the excess late-time brightness of the SN 2002hh echo. For the time being, SN $2002 \mathrm{hh}$ is unique - it appears to have the brightest echo yet observed around a SN, by a wide margin.

We would like to thank the referee for many useful suggestions. D. L. W. was supported by the Natural Sciences and Engineering Research Council of Canada (NSERC). This project was also supported by the NSF/REU grant AST 00-97694 and the Nantucket Maria Mitchell Association. We would like to thank Eric Feigelson at Pennsylvania State University for suggesting this as an interesting topic for investigation. Observations were obtained during programs GN-2004B-C-3, GN-2004B-DD-6, GN-2005B-Q-54, and GN-2006A-Q-52 at the Gemini Observatory, which is operated by AURA under a cooperative agreement with the NSF on behalf of the Gemini partnership. We are indebted to the Gemini Observatory and its staff for helping to obtain these data and also for providing DD time for further observations of SN 2002hh. This research used the facilities of the Canadian Astronomy Data Centre operated by the National Research Council of Canada with the support of the Canadian Space Agency.

\section{APPENDIX}

\section{TERTIARY STANDARDS FOR NGC 6946}

The frequency with which supernovae have appeared in NGC 6946 during the last century suggests that the definition of a local set of faint tertiary standards, suitable for use on large telescopes, would be a worthwhile undertaking. In $\S 2$, we described the determination of the transformations to the $V, R$, and $I$ system of local photometric standards list by Pozzo et al. (2006) in their Table 1 . We defined a set of fainter standards in the following way. Stars selected were present in our output photometry lists in all three filters $\left(g^{\prime}, r^{\prime}\right.$, and $\left.i^{\prime}\right)$ at all six epochs for which the photometric instrumental uncertainties were all less than or equal to 0.03 mag. There were 110 such stars. These are labeled 101-210 so that there is no confusion with the Pozzo et al. numbering scheme.

The instrumental $g^{\prime}, r^{\prime}$, and $i^{\prime}$ magnitudes were transformed to the VRI system determined by Pozzo et al. (2006), and the unweighted mean and standard deviation of the six points for each star are listed in Table 2 . The uncertainties do not formally include the uncertainties in the transformation - they are the standard deviations for the set of six transformed magnitudes in each filter. The locations of the standard stars are plotted in Figure 6, which is an $r^{\prime}$ image with an exposure of 60.0 s obtained with GMOS-N on 2004 August 20. North is $10^{\circ}$ counterclockwise from vertical, and east is $90^{\circ}$ clockwise from north. The image scale is $0.1454^{\prime \prime}$ per pixel, and the height and width of the image are $149^{\prime \prime}$ and $335^{\prime \prime}$, respectively. 
TABLE 2

Tertiary VRI Photometric Standards for NGC 6946

\begin{tabular}{|c|c|c|c|c|c|c|}
\hline Star & $\begin{array}{c}V \\
(\mathrm{mag})\end{array}$ & $\begin{array}{c}\sigma_{V} \\
(\mathrm{mag})\end{array}$ & $\begin{array}{c}R \\
(\mathrm{mag})\end{array}$ & $\begin{array}{c}\sigma_{R} \\
\text { (mag) }\end{array}$ & $\begin{array}{c}I \\
(\mathrm{mag})\end{array}$ & $\begin{array}{c}\sigma_{I} \\
(\mathrm{mag})\end{array}$ \\
\hline $1 .$. & 20.31 & 0.03 & 19.35 & 0.04 & 18.42 & 0.05 \\
\hline 02. & 18.71 & 0.02 & 18.07 & 0.02 & 17.46 & 0.03 \\
\hline & 20.14 & 0.01 & 19.23 & & 71 & 0.02 \\
\hline $104 \ldots$ & 20.89 & 0.03 & 20.30 & & & 0.10 \\
\hline $05 \ldots$ & 19.95 & & & & & 0.04 \\
\hline $06 \ldots$ & 17.82 & & & & & 0.02 \\
\hline $07 \ldots$. & & & & & & 0.08 \\
\hline $08 \ldots$ & & & & & & 0.04 \\
\hline $09 \ldots$. & 18.15 & 0.01 & 17.42 & 01 & 16.80 & 0.01 \\
\hline $10 \ldots \ldots \ldots$ & 19.80 & 0.01 & 19.07 & 00 & 40 & .01 \\
\hline $11 \ldots \ldots \ldots \ldots \ldots$ & 17.38 & 0.01 & 16.92 & 01 & 43 & .01 \\
\hline $12 \ldots$ & 20.74 & 0.02 & 20.39 & 02 & 20.02 & .03 \\
\hline $13 \ldots . .$. & 20.53 & 0.01 & 19.77 & .01 & 19.10 & 0.02 \\
\hline $14 \ldots$. & 19.86 & 0.01 & 19.09 & 0.02 & 18.40 & 0.02 \\
\hline$\ldots \ldots$. & 19.86 & 0.01 & 18.83 & 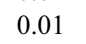 & 0 & 0.01 \\
\hline $16 \ldots \ldots \ldots \ldots$ & 20.29 & 0.00 & 22 & 01 & .09 & 0.01 \\
\hline 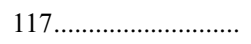 & 20.53 & 0.02 & 19.59 & & & 0.02 \\
\hline $18 \ldots \ldots \ldots \ldots \ldots \ldots \ldots \ldots \ldots \ldots \ldots \ldots \ldots \ldots \ldots . .$. & 19.15 & 0.01 & 3 & & & 0.01 \\
\hline $19 \ldots \ldots .$. & 20.29 & 0.01 & 63 & & & 0.03 \\
\hline 20 & 1743 & 0.01 & & & & 0.01 \\
\hline $21 \ldots$ & $1 c^{2}$ & $0 .($ & & & & 0.01 \\
\hline $122 .$. & 18 & 0.0 & & & & 0.01 \\
\hline $123 \ldots$ & 20 & 0. & 1 & ) & & .01 \\
\hline $124 \ldots$ & 18. & 0.01 & 1 & 2 & & 0.01 \\
\hline $125 \ldots$ & 17.57 & 0.01 & & 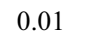 & & 0.01 \\
\hline $126 \ldots$ & 19. & 0.01 & & 1 & & .01 \\
\hline $127 \ldots$ & 19. & & & & & 01 \\
\hline $128 \ldots$ & 1 & & & & & 01 \\
\hline $129 \ldots$ & & & & & & 1 \\
\hline $130 \ldots$ & & & & & & 2 \\
\hline $131 \ldots$ & & & & & & 01 \\
\hline $32 \ldots$ & 2 & & & & & .01 \\
\hline $33 \ldots \ldots$. & 18 & 0. & & 1 & 6 & .01 \\
\hline $134 \ldots \ldots$. & 18 & 0.0 & 7 & 0 & 17. & .01 \\
\hline $35 \ldots \ldots$ & 20.77 & 0.04 & 3 & 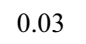 & 19.2 & 0.03 \\
\hline $136 \ldots$ & 20.98 & 0.01 & 20.33 & .01 & 19.72 & 0.02 \\
\hline$\ldots \ldots$. & 20.10 & 0.01 & 38 & 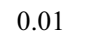 & 18.7 & 0.02 \\
\hline $38 \ldots$. & 19.75 & 0.01 & 20 & 0.01 & 18.65 & 0.01 \\
\hline $139 \ldots \ldots \ldots \ldots \ldots \ldots \ldots \ldots$ & 17.71 & 0.01 & 17.15 & 0.01 & 16.62 & 0.01 \\
\hline $140 \ldots \ldots \ldots \ldots \ldots \ldots \ldots$ & 18.68 & 0.01 & 18.19 & & 17.70 & 0.01 \\
\hline $141 \ldots \ldots \ldots \ldots \ldots \ldots \ldots$ & 20.21 & 0.02 & 19.18 & 0.01 & 18.19 & 0.01 \\
\hline $142 \ldots$ & 19.3 & 0.00 & & & & 0.00 \\
\hline $143 \ldots$ & 19.25 & 0.01 & 18.67 & 0.01 & 18.09 & 0.01 \\
\hline $144 \ldots$. & 19.1 & 0.0 & 18.62 & 0 & 10 & 0.01 \\
\hline $145 \ldots \ldots \ldots$ & 17.67 & 0.0 & 17.16 & 1 & 16.67 & 0.01 \\
\hline $146 \ldots$ & 18.8 & 0.0 & 18 & 0.01 & 17.75 & 0.01 \\
\hline $147 \ldots$ & 18. & 0.0 & & 0. & & 0.01 \\
\hline $148 \ldots$ & 20. & 0. & & 0. & & 0.01 \\
\hline $149 \ldots$ & & & & & & .00 \\
\hline $150 \ldots$ & & & & & & .01 \\
\hline $151 \ldots$ & & & & & & .00 \\
\hline $152 \ldots$ & 20.62 & & & & & 0.02 \\
\hline $153 \ldots$ & 17.44 & 0.01 & 16.77 & 0.00 & 16.12 & 0.01 \\
\hline & 20.94 & 0.03 & 20.08 & 0.04 & 19.29 & 0.05 \\
\hline $155 \ldots \ldots \ldots$ & 20.70 & 0.01 & 19.59 & 0.01 & 18.54 & 0.01 \\
\hline
\end{tabular}

TABLE 2-Continued

\begin{tabular}{|c|c|c|c|c|c|c|}
\hline Star & $\begin{array}{c}V \\
(\mathrm{mag})\end{array}$ & $\begin{array}{c}\sigma_{V} \\
(\mathrm{mag})\end{array}$ & $\begin{array}{c}R \\
(\mathrm{mag})\end{array}$ & $\begin{array}{c}\sigma_{R} \\
(\mathrm{mag})\end{array}$ & $\begin{array}{c}I \\
(\mathrm{mag})\end{array}$ & $\begin{array}{c}\sigma_{I} \\
(\mathrm{mag})\end{array}$ \\
\hline $156 \ldots \ldots \ldots$ & 21.03 & 0.01 & 20.43 & 0.03 & 20.32 & 0.03 \\
\hline $157 \ldots \ldots \ldots \ldots \ldots \ldots$ & 19.66 & 0.02 & 18.77 & 0.01 & 18.01 & 0.01 \\
\hline $158 \ldots \ldots \ldots \ldots \ldots \ldots$ & 19.54 & 0.02 & 18.66 & 0.00 & 16.87 & 0.01 \\
\hline $159 .$. & 18.07 & 0.01 & 17.43 & 0.00 & 16.85 & 0.00 \\
\hline $160 \ldots$. & 18.46 & 0.01 & 17.89 & 0.01 & 17.33 & 0.01 \\
\hline 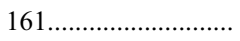 & 19.98 & 0.01 & 19.27 & 0.01 & 18.64 & 0.01 \\
\hline $162 \ldots \ldots$ & 19.81 & 0.01 & 18.74 & 0.01 & 17.38 & 0.00 \\
\hline 163. & 19.60 & 0.02 & 18.88 & 0.01 & 18.21 & 0.01 \\
\hline $164 \ldots$ & 17.87 & 0.01 & 17.14 & 0.01 & 16.49 & 0.01 \\
\hline $165 \ldots$ & 20.83 & 0.01 & 20.18 & 0.02 & 19.58 & 0.02 \\
\hline $166 \ldots \ldots$ & 20.54 & 0.01 & 19.84 & 0.00 & & 0.01 \\
\hline $167 \ldots \ldots$ & 20.69 & 0.02 & 19.72 & 0.01 & 80 & 0.01 \\
\hline $168 \ldots \ldots \ldots$ & 20.67 & 0.03 & 19.59 & 0.01 & 63 & 0.01 \\
\hline $169 \ldots \ldots$ & 18.61 & 0.00 & 18.08 & 0.00 & & 0.01 \\
\hline $170 \ldots$. & 19.11 & 0.01 & 18.48 & 0.01 & 17.86 & 0.01 \\
\hline $171 \ldots \ldots \ldots \ldots \ldots$ & 20.67 & 0.02 & 20.30 & 0.02 & 19.82 & 0.03 \\
\hline $172 \ldots \ldots$ & 17.18 & 0.01 & 16.43 & 0.01 & 15.80 & 0.01 \\
\hline $173 \ldots \ldots \ldots \ldots \ldots \ldots \ldots \ldots \ldots \ldots \ldots \ldots$ & 18.76 & 0.01 & 18.10 & 0.01 & 17.47 & 0.01 \\
\hline $174 \ldots \ldots \ldots$ & 17.71 & 0.01 & 17.02 & 0.00 & 16.34 & 0.01 \\
\hline $175 \ldots \ldots$ & 20.37 & 0.03 & 19.51 & 0.01 & 18.76 & 0.03 \\
\hline $176 \ldots \ldots$ & 21.36 & 0.03 & 20.57 & 0.01 & 19.88 & 0.02 \\
\hline $177 \ldots \ldots$ & 17.81 & 0.01 & 17.29 & 0.00 & 16.76 & 0.00 \\
\hline 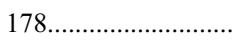 & 18.46 & 0.01 & 17.83 & 0.01 & 17.23 & 0.01 \\
\hline 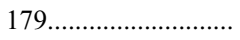 & 18.84 & 0.01 & 18.16 & 0.01 & 17.52 & 0.01 \\
\hline $180 \ldots \ldots \ldots \ldots \ldots \ldots$ & 19.65 & 0.01 & 18.87 & 0.02 & 18.25 & 0.01 \\
\hline $181 \ldots$ & 19.62 & 0.01 & 18.99 & 0.01 & 18.39 & 0.02 \\
\hline $182 .$. & 18.96 & 0.01 & 18.48 & 0.01 & 17.97 & 0.01 \\
\hline $183 \ldots \ldots \ldots$ & 21.13 & 0.03 & 20.32 & 0.02 & 19.64 & 0.01 \\
\hline $184 \ldots \ldots$ & 20.54 & 0.04 & 19.61 & 0.03 & 18.81 & 0.02 \\
\hline $185 \ldots$. & 20.66 & 0.02 & 20.30 & 0.03 & 19.81 & 0.04 \\
\hline $186 \ldots \ldots .$. & 20.29 & 0.02 & 19.31 & 0.02 & 17.78 & 0.02 \\
\hline $187 \ldots$ & 17.98 & 0.01 & 17.09 & 0.01 & 16.30 & 0.01 \\
\hline $188 \ldots$ & 19.80 & 0.02 & 18.76 & 0.01 & 17.54 & 0.01 \\
\hline $189 \ldots \ldots$ & 16.91 & 0.01 & 16.34 & 0.01 & 15.76 & 0.01 \\
\hline $190 \ldots$ & 19.94 & 0. & 18 & 0 & 17 & .02 \\
\hline $191 \ldots$ & 19.49 & 0 & 1 & .01 & 10 & .01 \\
\hline $192 \ldots \ldots \ldots$ & 20.58 & 0.03 & 19.59 & 0.02 & 29 & 0.02 \\
\hline $193 \ldots \ldots$. & 19.49 & 0.01 & 18.84 & 0.01 & 18.22 & 0.01 \\
\hline $194 \ldots \ldots \ldots$ & 20.24 & 0.02 & 19.48 & 0.01 & 18.78 & 0.02 \\
\hline $195 \ldots \ldots \ldots \ldots \ldots \ldots$ & 19.05 & 0.01 & 18.31 & 0.00 & 17.63 & 0.01 \\
\hline $196 \ldots \ldots . .$. & 17.56 & 0.01 & 16.89 & 0.01 & 16.28 & 0.02 \\
\hline $197 \ldots$ & 17.99 & 0.01 & 17.41 & 0.01 & 16.89 & 0.02 \\
\hline $198 \ldots \ldots$. & 20.17 & 0.01 & 19.53 & 0.00 & 18.92 & 0.02 \\
\hline $199 \ldots \ldots . .$. & 19.11 & 0.01 & 18.29 & 0.01 & 17.57 & 0.02 \\
\hline $200 \ldots \ldots \ldots \ldots \ldots \ldots$ & 18.80 & 0.01 & 18.27 & 0.01 & 17.71 & 0.02 \\
\hline $201 \ldots \ldots \ldots \ldots \ldots \ldots \ldots$ & 20.68 & 0.02 & 20.08 & 0.02 & 19.47 & 0.06 \\
\hline $202 \ldots \ldots \ldots \ldots$ & 20.70 & 0.02 & 20.18 & 0.01 & 19.64 & 0.02 \\
\hline $203 \ldots \ldots$ & 20.20 & 0.01 & 19.40 & 0.01 & 18.70 & 0.02 \\
\hline $204 \ldots \ldots$ & 19.40 & 0.01 & 18.50 & 0.01 & 17.70 & 0.02 \\
\hline $205 \ldots \ldots \ldots$ & 21.05 & 0.05 & 20.44 & 0.05 & 20.03 & 0.04 \\
\hline $206 \ldots \ldots \ldots \ldots \ldots \ldots$ & 16.98 & 0.01 & 16.46 & 0.01 & 15.94 & 0.01 \\
\hline $207 \ldots$ & 19.05 & 0.01 & 18.40 & 0.01 & 17.77 & 0.02 \\
\hline $208 \ldots$ & 18.91 & 0.01 & 18.34 & 0.01 & 17.76 & 0.01 \\
\hline $209 .$. & 21.21 & 0.04 & 20.52 & 0.03 & 19.86 & 0.02 \\
\hline $210 \ldots \ldots \ldots \ldots \ldots \ldots \ldots \ldots$ & 20.32 & 0.09 & 19.36 & 0.07 & 18.59 & 0.06 \\
\hline
\end{tabular}




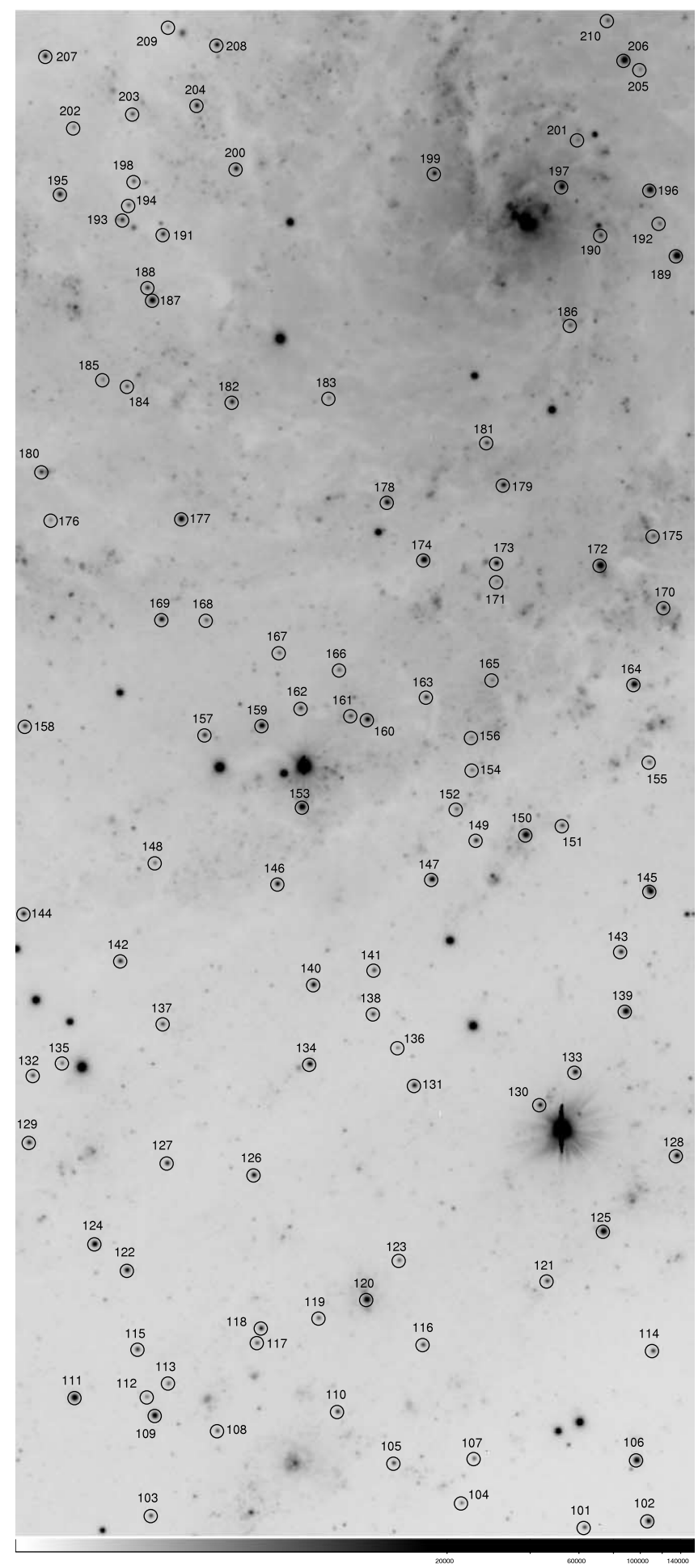

FIG. 6.-Stars included in Table 2 as tertiary standards. 
Barlow, M. J., et al. 2005, ApJ, 627, L113

Caldwell, J. A. R., et al. 1993, MNRAS, 262, 313

Danziger, I. J., Lucy, L. B., Bouchet, P., \& Gouiffes, C. 1991, in Supernovae: The Tenth Santa Cruz Workshop in Astronomy and Astrophysics, ed. S. E. Woosley (Springer: New York), 69

Duschinger, M., Puls, J., Branch, D., Hoeflich, P., \& Gabler, A. 1995, A\&A, 297, 802

Elmhamdi, A., et al. 2003, MNRAS, 338, 939

Filippenko, A. V., Foley, R. J., \& Swift, B. 2002, IAU Circ. 8007, 2

Gerardy, C. L., Fesen, R. A., Höflich, P., \& Wheeler, J. C. 2000, AJ, 119, 2968

Hamuy, M., \& Suntzeff, N. B. 1990, AJ, 99, 1146

Li, W. 2002, IAU Circ. 8005, 1

Lucy, L. B., Danziger, I. J., Gouiffes, C., \& Bouchet, P. 1989, in IAU Colloq. 120, Structure and Dynamics of the Interstellar Medium, ed. G. Tenorio-Tagle, M. Moles, \& J. Melnick (Lecture Notes in Physics 350; Berlin: Springer), 164 1991, in Supernovae: The Tenth Santa Cruz Workshop in Astronomy and Astrophysics, ed. S. E. Woosley (Springer: New York), 82

Mattila, S., \& Meikle, W. P. S. 2001, MNRAS, 324, 325

McGough, C., Clayton, G. C., Gordon, K. D., \& Wolff, M. J. 2005, ApJ, 624, 118

\section{REFERENCES}

Meikle, P., Mattila, S., Smartt, S., MacDonald, E., Clewley, L., \& Dalton, G. 2002, IAU Circ. 8024, 1

Meikle, W. P. S., et al. 2006, ApJ, 649, 332

Patat, F. 2005, MNRAS, 357, 1161

Patat, F., Benetti, S., Cappellaro, E., \& Turatto, M. 2006, MNRAS, 369, 1949

Phillips, M. M., \& Williams, R. E. 1991, in Supernovae: The Tenth Santa Cruz Workshop in Astronomy and Astrophysics, ed. S. E. Woosley (Springer: New York), 36

Pozzo, M., Meikle, W. P. S., Fassia, A., Geballe, T., Lundqvist, P., Chugai, N. N., \& Sollerman, J. 2004, MNRAS, 352, 457

Pozzo, M., et al. 2006, MNRAS, 368, 1169

Sahu, D. K., Anupama, G. C., Srividya, S., \& Muneer, S. 2006, MNRAS, 372, 1315

Sirianni, M., et al. 2005, PASP, 117, 1049

Sugerman, B. E. K. 2003, AJ, 126, 1939

Sugerman, B. E. K., et al. 2006, Science, 313, 196

Van Dyk, S. D., Li, W., \& Filippenko, A. V. 2006, PASP, 118, 351

Walker, A. R., \& Suntzeff, N. B. 1991, PASP, 103, 958

Wang, L. 2005, ApJ, 635, L33 